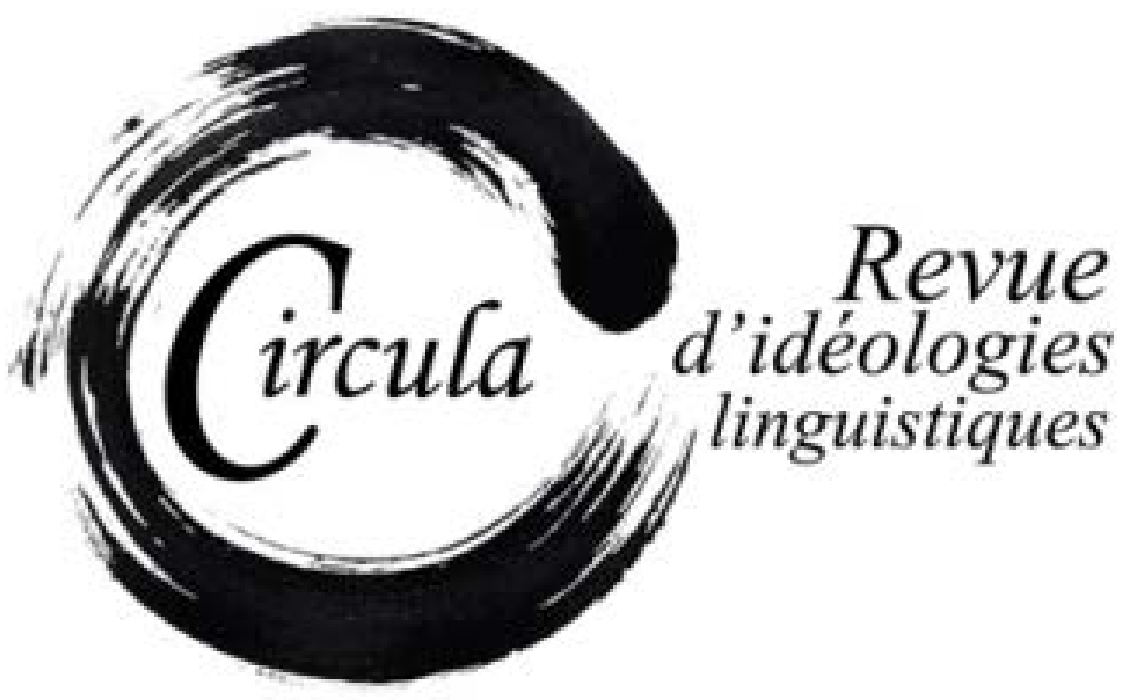

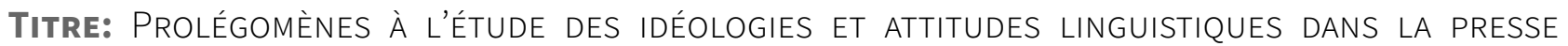
PÉRIODIQUE SOUS L'ANCIEN RÉGIME

Auteur(s): MAirI MCLAughlin, UniVERSity of CALIFORNIA, BERKELEY

Revue: Circula, NumÉro 1, PAGES 4-25

ISSN: 2369-6761

DiRECTEURS: Wim REMYSEN ET SABINE SCHWARZE

URI: HTTP://HDL.HANDLE.NET/11143/7993

DOI: $10.17118 / 11143 / 7993$ 


\title{
Prolégomènes à l'étude des idéologies et attitudes linguistiques dans la presse périodique sous l'Ancien Régime ${ }^{1}$
}

\author{
Mairi McLaughlin, University of California, Berkeley \\ mclaughlin@berkeley.edu
}

Résumé : Cet article examine si la presse a pu participer à la construction de l'idéologie et des attitudes linguistiques aux XVII et XVIII ${ }^{e}$ siècles. La recherche est fondée sur l'analyse d'un corpus de périodiques généralistes comprenant trois journaux d'actualité (la Gazette, la Gazette d’Amsterdam, le Journal de Paris), le Journal des savans et le Mercure galant. Nos analyses visent à déterminer si et comment ces supports médiatiques ont pu toucher l'image de la langue. Il est possible de comparer le discours métalinguistique de la presse pré-moderne à deux autres types de textes : les livres de remarques produits au siècle classique, et les chroniques de langue dans la presse moderne.

Mots-clés : histoire de la langue ; idéologie de la langue ; attitudes linguistiques; histoire de la presse ; remarqueurs

\begin{abstract}
This article investigates whether the French press contributed to the construction of language attitudes and ideologies in the 17 th and 18th centuries. The research is based on the analysis of a corpus of generalist periodicals. The corpus includes three newspapers (the Gazette, the Gazette d'Amsterdam, and the Journal de Paris) as well as the Journal des savans and the Mercure galant. In the analysis, I explore whether and how these different titles played a role in the formation of the image of the French language. I also suggest that the metalinguistic discourse of the French press from the 17th and 18th centuries should be considered in relation to two other text types: books of "remarks" written in 17th-century France, and the language columns published by newspapers in the 20th and 21st centuries.
\end{abstract}

Keywords: history of French; language ideology; linguistic attitudes; history of the press; the "remarqueurs"

1. Nous remercions Wendy Ayres-Bennett, Olivia Walsh et les lecteurs anonymes pour leurs commentaires détaillés sur une première version de cet article. 


\section{Introduction}

Cet article s'inscrit dans le contexte plus large d'un projet concernant les origines et l'évolution du français journalistique ${ }^{2}$. Le développement de la sociolinguistique historique a mené à une diversification des types de textes utilisés dans la recherche portant sur l'histoire du français ${ }^{3}$, mais nous n'avons trouvé que quelques analyses ponctuelles qui s'intéressent à la langue de la presse historique. Citons, à titre d'exemple, les travaux de Ayres-Bennett (2004 : 82-108) et de Lodge (2004 : 171-190) sur les mazarinades, ainsi qu'une analyse courte mais révélatrice d'un extrait du Mercure de France publié en 1732 (Ayres-Bennett, 1996 : 207-211). L'absence d'études approfondies de la presse historique de langue française constitue une lacune sérieuse dans la linguistique française. Notons, d'une part, qu'un contraste net apparaît entre la linguistique historique du français et la linguistique historique d'autres langues européennes parce que de nombreux travaux ont déjà été consacrés à l'étude de la langue de la presse historique de langue anglaise ${ }^{4}$. D’autre part, la langue des médias contemporains est une importante source de données linguistiques utilisées par les chercheurs travaillant sur le français contemporain ${ }^{5}$. Dans ce contexte, l'objectif de notre projet est de fournir la première étude systématique de la langue utilisée dans la presse historique de langue française.

Le présent article a pour but d'étudier le rôle éventuel de la presse historique dans la construction et l'évolution des idéologies et attitudes linguistiques aux XVII et XVIII siècles. Les histoires de la langue ont en commun d'accorder une place importante aux discours métalinguistiques qui circulaient à l'époque ; ces discours ont contribué à la construction d’idéologies et d'attitudes linguistiques concernant le français standard, les variétés du français, et les autres langues. Comme le note Seguin (1999: 227), les époques classique et post-classique ont «fortement contribué à former l'image que nous avons encore de notre langue et à imprimer à celle-ci un caractère qui la distingue de celles des pays avoisinants : nulle part un mouvement aussi soutenu n'a porté vers l'uniformisation et la recherche de règles suffisamment nettes ». La présente étude se place dans le contexte général de la recherche sur les idéologies et attitudes linguistiques du passé6. Citons, pour la période pré-moderne, la recherche centrée sur le rôle joué par les remarqueurs (Ayres-Bennett, 1987 ; Caron, 2004 ; Ayres-Bennett et Seijido, 2011). La méthodologie que nous employons dans la présente étude est similaire à celle décrite par Sairio et Pallander-Collin (2012) qui s'intéressent à la reconstruction

2. Voir McLaughlin (à paraître) ; le projet de recherche intitulé «The origins and evolution of journalistic French » est financé par une bourse du Hellman Fellows Fund (2012-2014).

3. Deux volumes ont paru récemment traitant de l'histoire du français sous cette perspective (Aquino-Weber, Cotelli et Kristol, 2009 ; Pooley et Largorgette, 2011).

4. Voir, par exemple, les actes du colloque CHINED sur le discours journalistique historique (Brownlees, 2006 ; Jucker, 2009).

5. Voir, par exemple, le projet et la série de colloques intitulés Le Français parlé des médias (http://www.aston.ac.uk/lss/ news/events/francais-parle-dans-les-medias/francais-parle-dans-les-medias-versionfranaise/) ou un domaine de recherche comme celui concernant l'usage des temps verbaux en français.

6. Pour un résumé d'études récentes qui offre un survol des différentes méthodes utilisées dans la recherche contemporaine, voir Preston (2013). 
des hiérarchies linguistiques du passé. Leur approche repose sur la multidisciplinarité : c'est l'histoire qui permet de déterminer quels groupes sociaux étaient prestigieux à une époque donnée, et c'est l'analyse des textes contemporains qui permet de découvrir les attitudes et idéologies (Sairio et Pallander-Collin, 2012 : 636). L'étude se situe aussi dans le contexte des nombreux travaux sur la rubrique des chroniques de langage dans la presse francophone ${ }^{7}$, même si la rubrique telle que la définit Remysen (2005) n'existe pas aux XVII et XVIII ${ }^{e}$ siècles.

Il importe de noter ici que les historiens n'ont pas manqué de porter leur attention sur la première publication périodique consacrée à la langue française elle-même, le Journal de la langue françoise, soit exacte soit ornée, fondé en 1784 par Urbain Domergue (1745-1810) ${ }^{8}$. Grâce en partie au travail de Busse et Dougnac (1992), l'importance de sa contribution à l'évolution des idéologies et des attitudes linguistiques est bien documentée ${ }^{9}$. Ce n'est pas pour rien que Domergue est considéré, aujourd'hui comme à l'époque, comme le « grammairien patriote » de la Révolution. Busse et Dougnac ont expliqué le rôle joué par le périodique lui-même en soulignant sa contribution à la vulgarisation du savoir parce que Domergue était « le seul grammairien sous la Révolution à servir d’intermédiaire entre les dirigeants politiques et la masse des citoyens pour porter à la connaissance de ceux-ci les problèmes linguistiques auxquels se heurtaient les premiers » (Busse et Dougnac, 1992 : 14-15). Ils expliquent que le périodique a surtout aidé ceux qui habitent dans les provinces à « se défaire des régionalismes pour avoir accès au bon usage du français » (Busse et Dougnac, 1992 : 56), et qu'il a promu le développement de néologismes, tels que sa fameuse invention loyaume pour remplacer le terme qui dénotait l'ancienne notion de royaume (Busse et Dougnac, 1992 : 98-99). Notre étude se situe dans la lignée des travaux sur le Journal de la langue françoise, mais la période couverte est antérieure à sa fondation. Aussi vise-t-elle à élargir le terrain de recherche en examinant les contributions éventuelles d'un certain nombre de sous-genres journalistiques qui ont précédé la parution de la première revue spécialisée consacrée à la langue elle-même.

Si cet article s'inscrit dans le cadre de travaux sur les attitudes linguistiques (historiques), l'approche qu'il adopte repose sur une combinaison de méthodes tirées de ce domaine et de celui de la linguistique historique. Plus précisément, nous profitons du corpus de publications périodiques historiques que nous avons construit pour notre projet sur les origines et l'évolution du discours journalistique historique. Le projet commence en 1631 parce que c'est la date de parution du premier périodique de langue française à avoir connu un succès à long terme ; il s'agit de la Gazette (de France) dont le premier numéro a été imprimé à Paris le 30 mai $^{10}$. Le projet se termine avec la Révolution en 1789

7. Citons, à titre d'exemple, l'article classique de Cellard (1983) ainsi que les travaux plus récents de Bochnakowa (2005, 2013). Les études de Remysen, qui portent pour leur part sur la presse canadienne, présentent l'avantage d'adopter une perspective diachronique et de remonter au XIXe siècle (par exemple, Remysen, 2012).

8. Ce titre a été publié pendant une période de 11 années (entre le $1^{\text {er }}$ septembre 1784 et le 7 août 1795), mais sa publication a été interrompue à plusieurs reprises ; au total, 166 numéros sont disponibles aujourd'hui. Pour plus d'informations, voir Dougnac (1991).

9. Voir aussi Choi (2007).

10. Une poignée de publications périodiques de langue française précède la Gazette, mais aucun de ces autres titres n’a 
à cause des changements politico-sociaux qui ont bouleversé le système médiatique français de manière définitive. Le corpus que nous avons constitué est présenté dans le Tableau 1.

Tableau 1 : Corpus analysé ${ }^{11}$

\begin{tabular}{|c|c|c|c|c|c|c|c|c|}
\hline Titre & $\begin{array}{c}\text { Année de } \\
\text { fondation }\end{array}$ & $\mathbf{1 6 3 2}$ & $\mathbf{1 6 5 7}$ & $\mathbf{1 6 8 2}$ & $\mathbf{1 7 0 7}$ & $\mathbf{1 7 3 2}$ & $\mathbf{1 7 5 7}$ & $\mathbf{1 7 8 2}$ \\
\hline Gazette & 1631 & jan & mar & mai & jui & sep & nov & jan \\
\hline Journal des savans & 1665 & & & mai & jui & sep & nov & jan \\
\hline Mercure galant & 1672 & & & mai & jui & sep & nov & jan \\
\hline Gazette d'Amsterdam & 1688 & & & & jui & sep & nov & jan \\
\hline Journal de Paris & 1777 & & & & & & & jan \\
\hline
\end{tabular}

Il se compose de cinq titres représentant des genres périodiques différents. La Gazette et la Gazette d'Amsterdam sont toutes deux des journaux d'actualité qui publient de courtes dépêches écrites par des correspondants et qui rappellent les dépêches publiées par les agences de presse de nos jours. Le Journal des savans, en revanche, s'occupe du monde du livre. Il offre des comptes rendus détaillés d'ouvrages scientifiques, dans son acception la plus large. Le Mercure galant offre ce que Vincent (2005 : 9) appelle « un contrepoint léger » aux gazettes et au Journal des savans « en jetant sur les événements un regard curieux et parfois ludique». Le Journal de Paris, quant à lui, est le premier quotidien de langue française et il présente un mélange de types de textes journalistiques. On peut le considérer comme l'ancêtre des journaux d'actualité contemporains. Comme l'indique le Tableau 1, ces cinq publications ont été fondées pendant la période qui nous intéresse et elles circulaient encore à la fin de la période sous examen.

Nous avons employé deux outils conceptuels pour assurer que le corpus constitue un échantillon représentatif de ces cinq périodes historiques qui peut être analysé en détail. La notion de « génération » a permis de choisir un an par tranche de 25 ans $^{12}$. L'échantillon commence en 1632, année pour laquelle il existe une série complète de numéros de la Gazette. La notion d'« année construite » (développée à partir de celle de «semaine construite » issue des études des médias ${ }^{13}$ ) a ensuite permis de choisir un mois différent pour chaque année. Pour chacun des mois inclus dans le corpus, nous

connu le succès qu'a eu la Gazette de Renaudot. Pour plus d'informations, voir Feyel (1999, 2000).

11. À cause des histoires parfois complexes des différents périodiques, nous n'avons indiqué que la date de leur fondation mais tous les périodiques ont été publiés jusqu'à la fin de la période sous examen. Pour plus d'informations sur les différentes publications, voir les articles qui leur sont consacrés dans le Dictionnaire des journaux (Sgard, 1991).

12. La discussion du découpage de la langue en tranches correspondant à des générations différentes par Ayres-Bennett et Seijido (2011 : 269-270) illustre la variété des définitions de la notion de " génération ». L'intervalle de 25 ans que nous avons employé ici représente un minimum.

13. Voir par exemple Valenzuela et Correa (2009) qui utilisent la semaine construite pour examiner la représentation genrée des candidat(e)s aux élections présidentielles au Chili. 
prenons en compte tous les numéros de chaque périodique ${ }^{14}$. Nous avons transcrit tous les numéros pour les mois indiqués en caractères gras : il s’agit des premiers et derniers numéros de chaque périodique dans l'échantillon, ainsi que tous les numéros dans l'échantillon pour le Journal des savans et pour la Gazette d'Amsterdam ${ }^{15}$. Ces transcriptions facilitent l'analyse de certains périodiques, mais nous aurons aussi recours à d'autres ressources qui répertorient les articles publiés dans le Journal des savans et dans le Mercure galant ${ }^{16}$.

L'organisation de l'article tient compte des trois sous-genres périodiques présents dans le corpus. Une analyse de l'impact éventuel des journaux d'actualité est suivie par l'examen du Journal des savans. Avant de conclure, nous présentons les résultats de notre analyse du Mercure galant.

\section{Les journaux d'actualité : la Gazette, la Gazette d'Amsterdam et le Journal de Paris}

Grâce à nos lectures des journaux historiques, nous avons établi une liste de mots-clés à utiliser pour identifier dans les transcriptions des gazettes et du Journal de Paris les articles qui pourraient toucher aux idéologies et aux attitudes linguistiques. Les mots-clés les plus pertinents sont françois, langage et langue ${ }^{17}$. Il faut remarquer d'abord la relative rareté de références explicites à la langue dans les journaux d'actualité du passé. Par exemple, le mot langue n'apparaît que 5 fois dans tous les numéros de la Gazette d'Amsterdam du corpus, c'est-à-dire 0,03 fois par tranche de 1000 mots. Ce constat met immédiatement en évidence un contraste important entre les journaux pré-modernes et les journaux modernes qui ont parfois accordé une place importante à la rubrique de la chronique du langage ${ }^{18}$. Ce contraste s'explique par les nombreuses différences qui séparent les gazettes historiques des journaux contemporains. Au lieu d'envisager une filiation directe entre les gazettes de la période pré-révolutionnaire et les quotidiens contemporains, ce sont les dépêches d'agences contemporaines qui représentent les descendants des gazettes. Cela dit, nous avons identifié deux types de nouvelles et un type de textes que l'on peut lier aux attitudes linguistiques.

14. La longueur du Mercure galant et du Journal de Paris nous a conduite à un deuxième découpage pour ces deux titres, de nouveau grâce à la notion de « semaine construite ».

15. Nous remercions notre assistante de recherche, Jenelle Thomas, qui a transcrit les numéros du Mercure galant et de la Gazette pour l'année 1782. Son travail a été financé par une bourse du Diplomacy and Culture Colloquium (UC Berkeley) et par le groupe Early Modern Patterns financé par la University of California Humanities Research Institute.

16. Voir la partie de la bibliographie consacrée aux tables analytiques.

17. Parmi les autres mots-clés, citons le mot dialecte qui a paru une fois dans les journaux, et le mot patois qui n'était pas présent dans les journaux d'actualité mais qui est utilisé dans le discours citant accompagnant une citation du picard dans le Journal des savans (novembre 1757). Les noms de langue (hollondais, latin) et le mot traduction permettent surtout d'extraire des textes publicitaires discutés ci-dessous.

18. Il est à noter que l'importance de cette rubrique varie selon le titre et selon la période : Muller (2004) considère les années 1950 et 1960 comme «l'âge d'or » de ces descendants des remarqueurs dans la presse. 
Le premier type de dépêches traite des informations concernant les relations avec un pouvoir étranger. Quand il s'agit de rapporter un échange diplomatique, le journaliste précise souvent dans quelle langue l'interaction a eu lieu. Les premiers exemples de ce type dans notre corpus datent du tout début de la période :

(1) On ne sçait icy quelle chere faire à l'Ambassadeur de Pologne, qui est logé chez le Comte de Grombech. Il eut audiance de son Altesse le 20. du courant: sa premiere harangue fut en Latin, la seconde en François, qu'il continua le 22. (Gazette de France, 2 janvier $1632^{19}$ )

(2) CE cercle veut aussi entrer en l'alliance de Suede à l'imitation du precedent. Aldringer \& Foucre y commançoient à former une armée pour la pousser en l'Alsace : mais le Roy de Suede ayant envoyé vers la noblesse pour les faire declarer amis ou ennemis, ils ont en fin parlé François. (Gazette de France, Relations, 4 février 1632)

L'intérêt de ces exemples réside dans le fait qu'ils présentent la langue française comme langue de la diplomatie. Nous savons que la langue française avait déjà été présentée comme langue universelle bien avant le fameux Discours sur l'universalité de la langue française en 1783 de Rivarol, entre autres par Bouhours $(1671: 37-38)^{20}$. Néanmoins, ces exemples laissent supposer que la presse d'actualité a pu également jouer un rôle à côté du discours métalinguistique des remarqueurs. Les informations concernant les choix de langue dans le contexte de la rencontre diplomatique peuvent aussi constituer un commentaire afférent à la hiérarchie linguistique : en (1), le français est placé au même niveau que le latin, langue toujours fort prestigieuse en ce début du XVIII siècle. La présentation des nouvelles en (1) et (2) montre que le choix de langue est inclus dans la dépêche comme une information de premier plan et non pas comme une information accessoire. Ajouté au fait que de tels exemples ne sont pas rares dans le corpus, ce constat nous permet de souligner l'importance éventuelle des informations diplomatiques pour ce qui concerne l'image de la langue.

Un deuxième type d'informations qui peut être lié à la construction de l'image de la langue concerne les académies. Il est des cas qui sont similaires à (1) et (2) parce qu'ils traitent d'un choix de langue; cette fois-ci il s'agit de la langue utilisée dans le cadre d'un appel à soumissions. Dans l'exemple (3), par exemple, l'académie laisse aux candidats à choisir entre le latin et le français, ce qui indique que le latin est toujours associé aux sciences et à l'érudition :

\section{(3) PROGRAME de l'Academie Royale des Belles-Lettres, Sciences \& Arts.}

L'Academie ayant été obligée de reserver un des deux Prix de cette année, elle en propose encore deux aux Savans de l'Europe, qui seront distribuez le 25. Août 1733. Elle destine un de ces Prix à celui qui expliquera avec le plus de probabilité le Sisteme de la Circulation de

19. Nous suivons les conventions de transcription utilisées par Ayres-Bennett et Seijido (2011), ce qui veut dire, entre autres, que l'orthographe et la ponctuation de l'original sont conservées; les ß sont remplacés par ss; et l'usage de u/v et de i/j suit l'usage moderne (voir Ayres-Bennett et Seijido, 2011 : 17, note 1).

20. Nous remercions Wendy Ayres-Bennett d'avoir signalé l'intérêt de ce texte pour la présente étude. 
la Seve dans les Plantes, ou qui établira le mieux l'opinion contraire. L'autre est destiné à celui qui donnera l'explication la plus probable de la Nature de l'Air \& de ses Proprietez. II sera libre d'envoyer les Dissertations en François ou en Latin : On demande qu'elles soient écrites en Caracteres lisibles ; elles ne seront reçûës pour le concours que jusqu'au 1er. (Gazette d’Amsterdam, 16 septembre 1732)

Qui plus est, la Gazette d'Amsterdam a publié des résumés des activités de l'Académie française comme illustré en (4) :

(4) L’Academie Françoise célebra le même jour la Fête de ce Saint : Pendant la Messe, on chanta un Pseaume en Musique, \& l'Abbé du Rosnel prononça le Panégirique du Saint avec beaucoup d'éloquence. L'après-midi, l'Academie donna le Prix de Poësie à l'Abbé Segui, \& elle déclara qu'elle avoit reservé celui d'Eloquence pour l'année prochaine. (Gazette d'Amsterdam, 5 septembre 1732)

La place importante accordée aux informations concernant l'Académie française par la Gazette d'Amsterdam a pu être perçue comme une légitimation de cette institution de la langue. Par ailleurs, nous ne pouvons omettre de rappeler que la Gazette d'Amsterdam circulait en dehors de la France. Cela laisse supposer qu'elle aurait aussi pu contribuer à la propagation de l'idée de la gouvernance linguistique que représente l'Académie française dans d'autres pays européens ${ }^{21}$.

Il est également possible d'esquisser un lien éventuel entre les textes publicitaires dans les gazettes et la construction des attitudes linguistiques ${ }^{22}$. Les publicités commencent à paraître dans notre corpus à partir de 1782 dans la Gazette et elles y sont présentes dès le début dans la Gazette d'Amsterdam et dans le Journal de Paris. Elles se trouvent à la fin du journal après les dépêches et s'en distinguent aisément par leur mise en page ${ }^{23}$. Nous suggérons que les publicités ont pu toucher les attitudes linguistiques quand elles faisaient la promotion de livres qui étaient des traductions $(5,6)$ ou qui étaient publiés en plusieurs langues (7). De telles publicités sont très fréquentes dans la presse d'actualité au XVIII ${ }^{\text {siècle. }}$

(5) Pierre Husson, Marchand Libraire à la Haye, imprime actuellement les Lettres de Ciceron à ses Amis, traduites en François, le Latin à côté, suivant l'Edition de Gravins. (Gazette d'Amsterdam, 8 juillet 1707)

$\overline{21 .}$ Monroe $(1910: 3)$ ne doute pas que les nombreux appels à la création d'une académie linguistique de langue anglaise aient été inspirés par l'Académie française.

22. De nombreux chercheurs ont déjà signalé la proximité des genres journalistique et publicitaire, même dans la presse historique. Par exemple, ces deux types de textes sont traités dans le même chapitre dans le Handbook of Historical Sociolinguistics (Percy, 2012).

23. Les variantes typographiques comprennent le nombre de colonnes, la taille de police et l'usage des italiques. 
(6) Le même Libraire donnera aussi dans peu au Public le 7. \& le 8. vol. des Mille \& une Nuit, Contes Arabes, traduits en François par Mr. Galland, de l'Academie Royale des Inscriptions \& Médailles. (Gazette d'Amsterdam, 8 juillet 1707)

(7) Il se débite chez Th. \& J.A. CRAJENSCHOT, Libraires à Amsterdam, \& chez d'autres Libraires dans les principales Villes de ces Provinces, ainsi qu'en celles de Brabant \& la Flandre, l'utile \& agréable ALMANACH D’ETAT pour l'année 1782, tant en Français qu'en Hollandais. (Gazette d'Amsterdam, 8 janvier 1782)

Il est évident que la promotion de traductions peut influer sur l'évaluation du prestige des langues impliquées. Les historiens de la langue ont associé le développement des traductions vernaculaires des textes classiques au temps de la Renaissance à l'accession des langues vulgaires au statut de langue littéraire ${ }^{24}$. Un exemple comme (5) pourrait donc être lié à une sorte de maintien du prestige de la langue française qui est déjà reconnue comme une langue littéraire prestigieuse. La publicité en (6) est peut-être plus intéressante parce qu'elle promeut une traduction à partir d'une langue qui n'était que rarement traduite à cette époque ${ }^{25}$. Ici il ne s'agira pas d'altérer le statut du français comme langue cible mais plutôt l'image de la langue source, l'arabe, qui en émerge comme langue littéraire. En raison de son lieu d'impression, la Gazette d'Amsterdam contient régulièrement des publicités comme (7), où le livre promu est publié en français et en «Hollandais » ${ }^{26}$. L'effet d'une telle publicité est de mettre le français et le néerlandais au même niveau dans la hiérarchie linguistique.

Les premières informations concernant les belles-lettres en dehors de la presse savante stricto sen-

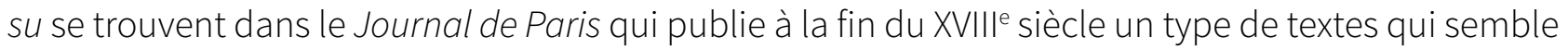
représenter un mélange du compte rendu et de la publicité (8) :

(8) ÉTRENNES du Parnasse. Choix de Poésies par M. le Prevost d'Exmes. A Paris, chez Couturier fils, Libraire, quai \& près l'Eglise des Augustins, au Coq. Petit in-12 d'environ 250 pages. [...]

Ce Recueil est suivi de différentes Traductions de Poésies étrangeres en vers François. L’Editeur (M. le Prevôt d'Exmes), a aussi inséré dans cet article des Pieces qui ont paru depuis longtems; il s'est ainsi ménagé les moyens de faire un choix plus agréable. On trouvera parmi ces traductions des morceaux déjà connus de Mrs François de Neufchateau, Blin de Sain-more, Harduin, de St. Ange, de la Harpe, \&c. Il y en a plusieurs qui paroissent pour la premiere fois, entre autres cette Fable imitée de l'Allemand, par M. de Monvel. Le fond est intéressant \& le style a la simplicité convenable à l'Apologue. (Journal de Paris, 21 janvier 1782)

\footnotetext{
24. Voir par exemple McLaughlin (2014).
}

25. Un certain nombre de textes scientifiques avaient été traduits de l'arabe en moyen français mais ce n'est qu'au XVIIIe siècle que commencera la traduction de textes arabes littéraires. Dotoli (2010 : 179) évoque une certaine mondialisation de la traduction au siècle des lumières : « Le XVIII ${ }^{e}$ siècle veut comprendre, et il traduit de toute matière et de tout monde. »

26. Le terme hollandais est utilisé plus souvent que flamand dans les publicités que nous avons examinées. 
Quand ils traitent de traductions, ces textes ont pu avoir des effets similaires à ceux produits par les publicités dans les gazettes. Il est à noter, en revanche, que c'est dans ce quotidien que l'on trouve les premiers commentaires dans la presse d'actualité qui traitent explicitement des variétés particulières de la langue française. En (8), par exemple, le style est loué pour sa simplicité et ailleurs, le journaliste fait l'éloge d'un livre pour enfants au nom de son « langage simple \& naïf » ${ }^{27}$. Ainsi s'illustre la capacité primitive qu'a eue la presse d'actualité de contribuer non pas seulement à la construction de l'image de la langue française dans son ensemble, mais aussi aux attitudes concernant ses variétés particulières, et surtout la variété de prestige.

Cette analyse d'un corpus de journaux d'actualité permet de constater que même sans la rubrique de la chronique du langage, la presse d'actualité a pu toucher les attitudes et idéologies linguistiques auX XVII et XVIII e siècles. La relative rareté de la thématisation de la langue empêche certes d'accorder à la presse historique un rôle aussi important que celui de la presse contemporaine qui, elle, semble pouvoir toucher simultanément plusieurs aspects de l'idéologie linguistique. Il paraît plutôt que la presse historique a pu participer à la construction et à la circulation d'une partie particulière de l'idéologie de la langue, à savoir l'image de la langue qui est centrée d'une part sur le rang élevé qu'elle occupe dans les hiérarchies linguistiques et d'autre part sur son universalité. Nous avons vu que cette image s'applique dans deux domaines spécifiques : la diplomatie internationale et le monde des sciences et de l'érudition. Les chercheurs tels que Milroy (2004: 166) ont déjà souligné l'importance de l'histoire dans l'établissement d'idéologies qu'elle considère être « profondément ancrées dans l'histoire » et « complètement naturalisées » (notre traduction). L'histoire du discours journalistique aide donc à expliquer pourquoi l'universalité de la langue française semble une évidence à la fin du XVIII e siècle car cette image de la langue a été promue par, entre autres, la presse dès le début du siècle classique. Si nous avons aussi insisté sur l'importance des informations concernant les académies, c'est parce que ce type d'informations a dû renforcer le prestige non pas de la langue elle-même, mais d'une des institutions clés de la standardisation de la langue aux XVIIe et XVIII 'e siècles, c'est-à-dire l'Académie française. De cette façon, on peut aussi lier les premiers journaux d'actualité à la construction et à la circulation de l'idéologie du standard en France : soutenir l'autorité c'est aussi soutenir le processus.

\section{Le Journal des savans}

Le Journal des savans est considéré comme une publication généraliste à cause de la vaste étendue de sujets couverts par ses comptes rendus qui concernent, entre autres, la théologie, l'astronomie, la médicine, l'architecture et l'histoire. Parmi tous ces sujets, certains sont plus pertinents que d'autres pour l'image de la langue. Nous proposons donc une analyse de l'éventuelle contribution de deux types de comptes rendus qui nous semblent les plus prometteurs: d'une part, les comptes rendus de recueils d'observations et de remarques sur la langue française et, d'autre part, les comptes rendus

27. Voir le Journal de Paris, 5 janvier 1782. 
de traductions. Nous nous sommes servie de la Table générale des matieres contenues dans le journal des savans (1759) afin de repérer les comptes rendus d'ouvrages traitant de livres de remarques entre 1665 et $1750^{28}$. L'analyse de comptes rendus de traductions se base sur la même Table générale mais porte également sur les numéros du Journal des savans inclus dans notre corpus (voir Tableau 1).

En lisant les comptes rendus des livres de remarques publiés entre la fondation du périodique et 1750, nous avons identifié plusieurs grands thèmes. Il est d'abord évident que l'utilité de ce type de textes métalinguistiques n'est jamais remise en question. Leur lecture est en effet présentée comme un élément essentiel du travail de celui qui aspire à la « pureté du stile » et au bon usage ${ }^{29}$. Dans un compte rendu des remarques d'Alemand (1688), le journaliste souligne cette nécessité en insistant sur l'incertitude qui règne en matière de langue - due en grande partie aux querelles entre commentateurs $^{30}$ - et valorise en même temps la pureté stylistique et le bon usage :

(9) Il semble même qu'il étoit nécessaire en ce tems-ci, où les contestations des Grammairiens sont arrivées à un point où l'on ne connoît presque plus l'état véritable de notre langue. La différence des sentimens que les uns \& les autres soutiennent avec opiniâtreté, jette dans des doutes embarrassans ceux qui souhaitent de parler \& d'écrire purement, \& qui pour cet effet cherchent le bon usage. (Journal des savans, 11 avril 1689)

Certains comptes rendus permettent de comprendre ce qui incite les lecteurs à lire les recueils de remarques : le plaisir qu'ils éprouvent et une certaine autosatisfaction sont tous deux illustrés par les commentaires du journaliste en 1726 dans un compte rendu des remarques de Malherbe (1725). Le journaliste commence par souligner dans une perspective comparative la difficulté du positionnement de l'adjectif en français :

(10) La Langue Gréque, la Langue Latine, \& la plupart des autres Langues laissent la liberté de placer l'adjectif devant ou après le substantif. II n'en est pas de même en François; \& c'est une difficulté considérable pour ceux qui apprennent cette Langue. (Journal des savans, février 1726)

Il explique ensuite l'intérêt qu'il y a à lire les remarques de Malherbe :

(11) Quoique M. Malherbe, qui traite cet article à la page 183, ne dise rien, que l'usage n'ait appris à un François, quelque ignorant qu'il soit d'ailleurs sur les regles de sa Langue ; c'est néanmoins avec un espéce de plaisir que l'on voit cet usage, auquel on est fait, réduit en prin-

28. La Table générale présente certaines autres catégories qu'il serait utile d'examiner dans une étude future. Citons, à titre d'exemple, les comptes rendus de livres traitant des origines des langues, des langues particulières et de l'Académie française.

29. Journal des savans, 4 août 1698.

30. Voir Ayres-Bennett et Seijido (2011 : 12-42) pour une discussion détaillée des rapports entre les principaux livres de remarques du siècle classique. 
cipes, \& que l'on s’apperçoit que malgré soi l'on parle bien, sans sçavoir pourquoi. (Journal des savans, février 1726)

Mais tout au long de la période, les éloges les plus flatteurs sont réservés pour le triumvirat de Vaugelas, Ménage et Bouhours et ceci même dans des comptes rendus d'ouvrages publiés par d'autres auteurs, comme l'illustre (12) où est reproduite la première phrase d'un compte rendu d'Aisy (1698) :

(12) Le meilleur moyen d'acquerir la connoissance de notre Langue est de bien étudier les remarques de M. de Vaugelas, celles de M. Menage, \& du P. Bouhours. (Journal des savans, 4 août 1698)

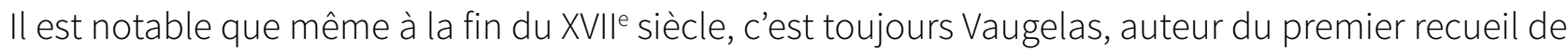
remarques, qui reçoit les éloges les plus enthousiastes : en 1690, par exemple, le journaliste écrit qu' «il suffit de dire que ces nouvelles Remarques sont de Mr. de Vaugelas, pour les faire estimer de tout le monde » (Journal des savans, 10 avril 1690). Ceci s'aligne bien sur notre conception contemporaine de l'ouvrage de Vaugelas : Ayres-Bennett et Seijido (2011 : 42), par exemple, lui accordent le statut de «modèle». Cela ne veut pourtant pas dire que les premiers livres de remarques sont lus sans critique. Les critiques concernent surtout un manque d'ordre dans les premiers recueils de remarques ${ }^{31}$; la

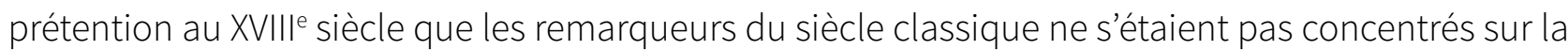
syntaxe $^{32}$; et la reconnaissance que la langue a changé depuis les premiers volumes de remarques, d'où le besoin de modifier certaines règles ${ }^{33}$.

En proposant un mélange d'évaluation critique et de promotion, les comptes rendus de livres de remarques ne faisaient pas que promouvoir les livres : grâce à leur apparition régulière dans le Journal des savans entre 1665 et 1750, ils ont dû aussi participer à la construction de l'image de la langue française et des attitudes envers ses variétés. Une analyse détaillée de leur contenu a montré surtout qu'ils auraient contribué à répandre l'idéologie de la langue qui tourne autour du bon usage, du mot juste et de la pureté du style. Ils ont aussi renforcé la légitimité des autorités linguistiques, à

31. Dans son compte rendu du Genie de la langue françoise, par exemple, le journaliste explique que " ce qui fait de la peine à ceux qui lisent ces trois auteurs [Vaugelas, Ménage et Bouhours], \& sur-tout aux étrangers, c'est qu'ils n'ont observé aucun ordre dans leurs remarques » (Journal des savans, 4 août 1698; voir aussi le Journal des savans, 16 avril 1685). Ayres-Bennett et Seijido (2011 : 43-44) considèrent le manque d’ordre comme un trait caractéristique du genre des remarques.

32. Voir par exemple un compte rendu d'Olivet (1738), où le journaliste note : «A l'occasion de ses deux vers, \& dans la vûë d'enrichir notre Syntaxe, que les remarques de Vaugelas, non plus que celles de Ménage \& du P. Bouhours, n'ont pas à beaucoup près épuisée; M. l'A. d'O. propose une régle fondamentale... » (Journal des savans, juillet 1738). Ayres-Bennett et Seijido (2011 : 140) donnent une image plus nuancée : selon leurs calculs, $40 \%$ des remarques de Vaugelas traitent de la syntaxe; le chiffre pour Bouhours se situe entre $18 \%$ et $25 \%$, tandis que « Ménage se démarque assez nettement en les développant peu ».

33. Le journaliste évoque le fait que la langue a changé parmi les nombreuses raisons qui expliquent l'intérêt des Nouvelles remarques de Mr. de Vaugelas publiées par Alemand (Vaugelas, 1690) : « Mr. Alemand a cru devoir faire des observations sur ces Remarques, à cause qu'il y a des décisions qui estoient bonnes au temps de Mr. de Vaugelas, \& qui ne le sont plus aujourd'hui » (Journal des savans, avril 1690). 
commencer par celle des remarqueurs et de leurs livres. II ne devrait pas surprendre que l'Académie française figure ici comme autorité responsable de remarques et d'autres publications métalinguistiques comme le dictionnaire (Académie française, 1694). La légitimité de l'Académie française comme autorité linguistique est explicitement construite dans un compte rendu des Remarques et decisions de l'Académie françoise recueillies par Tallemant (1698), où le journaliste fait remarquer que « La Reputation de l'Académie Françoise, donnera toujours un grand poids aux décisions qui paroîtront venir d'elle sur les doutes, \& sur les difficultés de la Langue » (Journal des savans, 15 décembre 1698). L'analyse des livres de remarques qu'offrent les journalistes a aussi renforcé l'autorité des grands auteurs parce qu'ils ont souvent choisi de commenter les sources utilisées par les remarqueurs. L'exemple le plus évident est un compte rendu des Remarques de grammaire sur Racine par Olivet (1738), où le journaliste explique et semble soutenir sa décision d'étudier « non des meilleures traductions en notre Langue », " mais ceux de nos Ouvrages François originaux, dont le mérite, depuis 50. ou 60. ans, est avoüé de tout le monde » (Journal des savans, juillet 1738).

Un examen de comptes rendus de traductions publiés par le Journal des savans a indiqué que ce ne sont pas que les comptes rendus de remarques qui ont pu participer à la propagation de l'idéologie du bon usage et des attitudes envers la pureté du style. En effet, les journalistes se servent des mêmes termes que les remarqueurs pour faire l'éloge des traductions. Ayres-Bennett et Seijido (2011 : 271) ont noté qu' " [à] partir de Vaugelas la plupart des remarqueurs mettent l'accent sur trois qualités essentielles : la pureté, la netteté et la propriété ». Dans l'exemple (13), par exemple, le journaliste loue la «manière aisée » et la «netteté du style » de la traduction de Cicéron par l'Abbé Gonthier:

(13) Quoy qu'on fasse fort difficilement parler François Ciceron, la maniere aisée \& la netteté du style avec lesquelles M. l'Abbé Gonthier a traduit cette piece ne luy fait rien perdre de sa beauté. (Journal des savans, 16 février 1682)

De même, l'exemple (14) inclut toute une série de termes (poli, juste, délicatesse, naïveté) qui s'associent eux aussi à la métalangue des remarqueurs :

(14) Comme la Grece n'a jamais rien eu de plus galand ni de plus poli que les poësies de Sapho \& d'Anacreon, nous pouvons dire que la France n'a guere rien veu de plus juste que cette traduction, tant par la delicatesse avec laquelle Mademoiselle le Fevre a imité dans cette copie la naïveté presque inimitable de l'original, que par le secret qu'elle a sceu trouver la premiere, de faire passer dans une prose fidelle toutes les graces que l'on trouve dans les vers Grecs. (Journal des savans, 16 février 1682)

Nous n'avons cité que deux exemples ici pour illustrer l'usage de ces termes, mais il en est certes d'autres. Nous voulons attirer l'attention sur ces termes parce qu'ils renforcent la conclusion que le Journal des savans a contribué à la circulation de l'idéologie de la langue développée dans l'œuvre des remarqueurs. Dans une étude future, il serait intéressant de développer cette piste en examinant des comptes rendus d'autres types de textes, comme les dictionnaires et les grammaires. II 
pourrait également être utile d'élargir le champ de l'enquête en tenant compte d'autres sous-genres périodiques tels que les « bibliographies ». Ces périodiques qui émergent au tournant du XVII siècle rendaient compte de livres publiés dans d'autres pays et pourraient être une source importante de commentaires concernant le style.

Avant de passer à l'analyse du Mercure galant, arrêtons-nous un instant sur l'impact éventuel non pas des commentaires des journalistes mais de la pratique éditoriale du Journal des savans en ce qui concerne la sélection des livres à passer en revue. Nous suggérons que tout comme les publicités diffusées dans les journaux d'actualité, les comptes rendus de traductions et d'ouvrages publiés dans des langues autres que le français auraient également pu toucher les attitudes linguistiques à un niveau plus général en affectant la construction des hiérarchies linguistiques de l'époque. Ailleurs, nous avons examiné la fréquence de différents types d'ouvrages dont rend compte le journaliste du Journal des savans entre 1682 et 1782 (McLaughlin, à paraître) : il s'agit d'ouvrages écrits en français, d'ouvrages traduits en français d'une autre langue (qu'elle soit morte ou vivante), et d'ouvrages non traduits écrits en une autre langue. II ressort de cette analyse que les ouvrages de langue française non traduits ne représentent guère plus de la moitié des comptes rendus ${ }^{34}$. Ce multilinguisme des ouvrages commentés par le Journal des savans découle de la nature même de l'entreprise du périodique dont le dessein explicite était de «faire sçavoir ce qui se passe de nouveau dans la Republique des lettres », un espace défini par le fait de dépasser les limites territoriales ("L'Imprimeur au lecteur », Journal des savans, 1665). Mais pour ce qui nous intéresse ici, il convient de souligner une diminution du nombre d'ouvrages en langues autres que le français passés en revue tout au long de la période sous examen, diminution qui s'accompagne d'une augmentation du nombre de traductions. Il est possible de lier la pratique de cette publication savante à la perception du statut de la langue française parmi les autres langues parce que la préférence pour les traductions qui

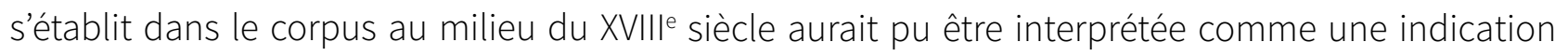
de l'importance de la langue française dans la République des lettres. Il semble en particulier que la réduction du nombre de comptes rendus d'ouvrages scientifiques écrits en latin aurait pu contribuer à l'affirmation du statut du français comme langue de science ${ }^{35}$.

En ce qui concerne la contribution des périodiques à la formation de l'idéologie linguistique, on peut constater un certain nombre de similarités générales entre les journaux d'actualité et le Journal des savans. C'est surtout dans la représentation du français comme langue scientifique qu'ils coïncident le plus. Les deux sous-genres périodiques partagent aussi le fait de renforcer le statut de certaines autorités linguistiques. Nous avons vu dans les deux cas des articles qui affirment l'autorité institutionnelle de l'Académie française en matière de langage. La contribution du Journal des savans paraît plus significative dans ce domaine parce que les comptes rendus de livres de remarques ont

34. Sur un total de 45 comptes rendus publiés aux mois de mai 1682, de juillet 1707, de septembre 1732, de novembre 1757 et de janvier 1782, 26 traitent d’ouvrages français non traduits. Pour plus de détails sur ces calculs, voir McLaughlin (à paraître).

35. Comme l'explique Siouffi (2007: 778), bien que le remplacement du latin dans le domaine scientifique ait commencé au XVI e siècle, ce processus ne s'est terminé qu'au XVIII siècle. 
aussi pour effet de souligner - parfois explicitement - l'autorité des remarqueurs eux-mêmes, ainsi que celle des auteurs et des sources métalinguistiques qu'ils invoquent. Mais dans cet article sur les attitudes et idéologies linguistiques, il est plus important de constater qu'en commentant les livres de remarques et les traductions, le Journal des savans a contribué à la propagation de l'idéologie linguistique des remarqueurs. Un travail récent par Ayres-Bennett et Seijido (2013) a montré que la pensée linguistique des remarqueurs doit faire l'objet d'une approche nuancée. Elles ont illustré l'hétérogénéité de leurs attitudes envers la seule notion du «bon usage » (Ayres-Bennett et Seijido, 2013 : 10). Au lieu de concevoir l'idéologie des remarqueurs comme une construction uniforme, nous préférons ici donc souligner les différents aspects de leur idéologie qui sont repris dans le Journal des savans. Dans les comptes rendus de traductions, nous avons identifié surtout la recirculation des qualités linguistiques prônées par les remarqueurs telles que la pureté, la délicatesse, la justesse, la propreté et la netteté. Les propos de Seguin (1999 : 227) cités ci-dessus soulignent l'importance de cette conceptualisation particulière du bon usage dans l'histoire de la langue française parce qu'elle reste au cœur de l'image de la langue française aujourd'hui. Les comptes rendus de livres de remarques, pour leur part, font la promotion de l'idéologie de la langue associée au bon usage en général. Il est donc clair que le Journal des savans a contribué à la propagation de l'idée de l'existence de formes correctes en matière de langage et qu'il a ainsi contribué à la diffusion de l'idéologie du standard mais de manière plus directe que ne l'a fait la presse d'actualité.

\section{Le Mercure galant}

Si le Mercure galant se distingue du Journal des savans de par son ton moins sérieux, il présente une diversité de matériaux comparable. Vincent (2005: 11) résume ainsi les grandes thématiques du périodique : «l'actualité d'intérêt public (champ où se joue la concurrence avec la Gazette et où apparaissent aussi le théâtre et le roman), la chronique familiale et mondaine, la vulgarisation scientifique, la musique et enfin la poésie ». II s'ensuit qu'on pourrait trouver des commentaires liés à la langue dans des discussions de plusieurs sujets différents. Nous nous concentrons donc ici sur deux types de sujets abordés dans le Mercure galant : la parution de livres de remarques et les questions linguistiques. Nous avons utilisé la table analytique de Vincent (1988) pour identifier les articles pertinents.

En dépit des différences formelles fondamentales entre le Journal des savans et le Mercure galant, il existe un chevauchement important entre leur traitement de nouvelles parutions dans le monde des livres. Tout comme le journaliste du Journal des savans, le journaliste du Mercure galant s'intéresse à la parution de recueils de remarques sur la langue française et lui aussi les présente sous une lumière positive. En 1672, par exemple, le journaliste du Mercure galant commente la parution des observations de Ménage (1672) et la citation (15) illustre le type d'éloges dont est accompagnée la parution de tels livres de remarques: 
(15) Quoy qu'on ne doive pas toûjours estimer un Ouvrage par son succés, on peut neantmoins juger du mérite de celuy-cy par le grand bruit qu'il fait, puis que c'est avec justice qu'il plaist ; \& je ne doute point que dans quelque temps, au lieu de dire parler Vaugelas, pour loüer ceux qui parleront bien, on ne dise parler Ménage. (Mercure galant, mai 1672)

Il est possible d'observer plusieurs similarités entre cet exemple et les comptes rendus publiés dans le Journal des savans. Il est d'abord évident que l'on peut lier de tels commentaires à la circulation de l'idéologie du bon usage, du « bien parler ». De même, le journaliste confère une légitimité aux autorités linguistiques, que ce soient des individus - Vaugelas, Ménage, Bouhours - ou bien leurs écrits.

II reste cependant certaines différences entre la présentation des ouvrages de remarques dans le Journal de savans et dans le Mercure galant. On observe surtout une certaine franchise dans les propos du journaliste du Mercure galant qui exprime ses opinions de manière claire et accessible. Ce trait est sans doute lié à une différence générique entre les deux périodiques car au départ, le Mercure galant s'adresse sous forme de lettre à une « Madame » anonyme habitant en province ${ }^{36}$; la forme épistolaire (factice) et la femme destinataire annoncent un ton plus léger ${ }^{37}$. De même, l'évaluation critique concernant, par exemple, la structuration des remarques n'est pas présente dans ce périodique et on peut supposer que c'est dû à son ton plutôt léger. Il convient de rappeler ici le travail de Vincent (2005 : 9) qui souligne l'importance de ce périodique qui, selon elle, « se révélera un incomparable déclencheur de relations intellectuelles ». Le style plus intime de ce périodique semble permettre au journaliste de participer de manière plus directe à la construction des attitudes et idéologies linguistiques. Ceci est bien illustré par l'exemple (15) parce que la manière directe dont le journaliste s'adresse à sa lectrice montre que ce dernier a cherché à peser sur la réception de l'ouvrage de ce remarqueur en l'infléchissant dans un sens positif. II note que c'est « avec justice qu'il plaist » et il termine son commentaire sur un ton exagéré en suggérant que Ménage va remplacer Vaugelas.

Ce ne sont pas que les discussions de textes métalinguistiques qui nous intéressent ici ; nous voulons également attirer l'attention sur les instances où le journaliste aborde des questions linguistiques de manière directe. Siouffi (2010 : 9) n'est pas le seul à avoir souligné l'importance de la conscience linguistique au siècle classique et ce fait expliquerait la présence d'un nombre non négligeable d'articles traitant de questions linguistiques dans ce périodique. Citons, à titre d'exemple, un petit « ouvrage » sur les « avantages » de la langue française publié dans un numéro du Mercure galant (octobre 1694). L'introduction fournie par le journaliste est reproduite en (16):

36. L'auteur s'adresse ainsi à la lectrice dans le premier numéro du Mercure galant: « Madame, Il n'estoit pas besoin de me faire souvenir que lors que vous partites de Paris, je vous promis de vous mander souvent des nouvelles capables de nourir la curiosité des plus Illustres de la Province qui doit avoir le bonheur de vous posseder si long-temps » (Mercure galant, janvier 1672).

37. Ayres-Bennett (2004: 120) décrit les traits stylistiques qui étaient censés convenir à un public féminin au XVIIe siècle : "In presenting specialist knowledge to a broader, female audience, it was considered important to avoid the possibility of boredom, by presenting the material in a clear but brief way, and shunning a pedantic tone and technical terminology ». 
(16) Le petit Ouvrage qui suit vous plaira sans doute, puis qu'il nous fait voir quels sont les avantages de nostre Langue, \& que vous en connoissez toutes les beautez, vous estant toujours attachée à bien parler, \& à bien écrire. (Mercure galant, octobre 1694)

Les propos du journaliste témoignent de sa capacité de commenter l'image de la langue dans ce périodique : il parle des « avantages » et des « beautez » de la langue. Le fait qu'il ait choisi de flatter sa lectrice en disant qu'elle a toujours voulu « bien parler » et «bien écrire » est aussi révélateur parce qu'il met en évidence la valeur associée au bon usage. Il est une partie de l'ouvrage qui nous concerne tout particulièrement ici parce que son auteur met l'accent explicitement sur l'universalité de la langue française:

(17) «L'étenduë de la Langue Françoise est digne de la noblesse de son origine. Elle passe les limites du Royaume. Elle ne se borne ny par les Pyrenées \& les Alpes, ny par le fleuve du Rhin. On entend le François dans toute l'Europe. La Langue Françoise a son Academie, le Tribunal de ses Juges en France ; mais elle a dans les autres Etats des Ecoles \& des Maistres qui l'enseignent; elle est connuë dans toutes les Cours, les Princes \& les Grands la parlent, les Ambassadeurs l'écrivent, \& le beau monde en fait une mode, \& un air de politesse. Elle merite d'estre ainsi universelle. » (Mercure galant, octobre 1694)

L'on voit s'articuler ici à la fin du siècle classique l'idéologie de langue française comme langue universelle. Cet exemple vient également soutenir nos propres observations concernant la description des échanges diplomatiques dans les gazettes (voir ci-dessus) parce qu'il lie explicitement l'usage du français dans la sphère diplomatique à son statut de langue universelle ${ }^{38}$. Notons enfin que l'auteur de l'ouvrage qui est reproduit dans le Mecure galant perçoit - et ainsi renforce - un lien entre l'Académie française et le prestige de la langue française, une observation non sans importance étant donné la fréquence avec laquelle le journaliste participe à la légitimation de l'Académie française dans les pages du périodique ${ }^{39}$. On ne peut omettre de faire remarquer ici que l'Académie française est un des rares thèmes qui réunissent tous les périodiques dans notre corpus.

Il est un deuxième type de discussions de questions linguistiques qu'il importe d'évoquer. Il s'agit de courts commentaires concernant l'usage douteux que l'on pourrait comparer à ceux des remarques publiées au XVII siècle, ou même aux chroniques de langage de la presse du XXe siècle. De tels commentaires ne sont pas rares dans le Mercure galant. À titre d'exemple, citons les titres suivants qui sont indiqués dans la table des matières des numéros particuliers : « Diamant brut ou diamant brute? » (Mercure galant, mai 1678) ; «Profil ou porfil ?» (Mercure galant, août 1678) ; «Prononciation du mot

38. Voir aussi une courte discussion de cet extrait dans le contexte de l'idée de l'universalité par Brunot (1917 : 137).

39. Ailleurs dans le Mercure, le journaliste loue le dictionnaire de l'Académie et il conseille son achat de manière directe à sa lectrice ("rien ne vous doit empescher de faire acheter son fameux dictionnaire ») et lui-même met l'accent sur son autorité ("Le Dictionnaire de l'Academie Françoise est un Ouvrage qui doit faire autorité », Mercure galant, janvier 1695). De même, l'auteur d'une histoire fait appel aux immortels pour servir d'arbitres lors d'une querelle violente concernant le participe passé du verbe paistre (Mercure galant, août 1680). Dans ce numéro, il dit qu'« ils sont reconnus pour les Maistres de la Langue, \& leur jugement sera sans appel » (Mercure galant, août 1680). 
"payen” » (Mercure galant, novembre 1679) ; "Question : doit-on écrire Bordeaux ou Bourdeaux? » (Mercure galant, janvier 1695). Ces discussions soulignent la capacité qu'avait la presse périodique déjà dans le premier siècle de son existence à jouer un rôle dans le processus de la standardisation de la langue française. Il est surtout frappant que l'usage douteux a été traité de manière similaire dans des publications qui appartiennent à des sous-genres distincts. S'il est vrai que dans de tels articles les journalistes se servent souvent de l'autorité de l'usage comme dernier arbitre, le fait même de traiter de doutes linguistiques implique la possibilité de les lever. Il est possible de comparer ces textes aux articles publiés un siècle plus tard par Domergue dans le Journal de la langue françoise. On peut également citer un article publié dans un des premiers numéros du Journal des savans qui discute du genre grammatical du mot comète (Journal des savans, 2 février 1665). Un examen du dernier paragraphe montre que la fonction de tels articles qui traitent de l'usage douteux ne se borne pas à illustrer le débat : ils essaient souvent de le conclure. Le journaliste prend le parti des modernes en insistant sur l'importance de l'usage et en rejetant l'idée qu'il faudrait rétablir le genre grammatical masculin du mot latin.

Cette analyse a mis en évidence des similarités entre le Mercure galant et les autres périodiques sous examen ici en ce qui concerne les différents aspects de l'idéologie de la langue qui sont touchés par le matériel qu'ils publient. Le fait que les auteurs du Mercure galant s'intéressent eux aussi aux livres de remarques veut dire que ce périodique peut être lié, tout comme le Journal des savans, à la diffusion de l'idéologie linguistique des remarqueurs. Le ton plus conversationnel du Mercure galant veut dire qu'on peut l'associer à la diffusion d'une idéologie plus directe et simple qui tourne autour de l'idée du bon usage. Non seulement les critiques de certains aspects des livres de remarques sontelles absentes, mais les auteurs insistent à plusieurs reprises sur la valeur du bien parler et du bien écrire en général. Le Mercure galant s'est aussi avéré similaire aux autres périodiques en ce qu'il a la capacité de construire l'autorité d'institutions linguistiques comme l'Académie française. De même a-t-on trouvé un exemple où le caractère universel de la langue est souligné dans le Mercure galant, tout comme il l'est dans les journaux d'actualité, quoique de manière moins explicite. Mais le Mercure galant se distingue des autres périodiques par la présence de deux types d'articles particuliers: ceux qui thématisent la langue même et ceux qui traitent de doutes ou de difficultés linguistiques. L'importance de ces deux types d'articles pour la construction et l'évolution de l'idéologie de la langue émerge clairement de la comparaison entre les trois sous-genres journalistiques examinés ici. L'exemple (17) illustre la richesse des commentaires sur la langue qui touchent des aspects fondamentaux de son idéologie : le prestige de ses origines, sa gouvernance par l'Académie française et son universalité. Les articles traitant de doutes et de difficultés sont importants eux aussi parce que leur existence signale l'importance de l'usage des formes correctes. De tels articles contribuent donc à la fois à la promotion de l'idéologie du standard et à la standardisation elle-même en diffusant des opinions et jugements. De la perspective de l'histoire des textes métalinguistiques, nous suggérons enfin que ces articles devraient être considérés comme une étape importante dans l'histoire de ce que Siouffi (2004: 9-10) appelle le « journalisme grammatical ». La place du Journal de la langue françoise (1784-1795) dans la lignée de textes qui vont des remarques jusqu'aux chroniques du langage 
a déjà été reconnue par Ayres-Bennett et Seijido (2011 : 263). Nous suggérons ici qu'il est possible d'interpréter les articles traitant de doutes et de difficultés dans une revue telle que le Mercure galant comme l'antécédent de la pratique d'Urbain Domergue.

\section{Conclusion}

Le but de cet article était de déterminer si et comment la presse de langue française a pu toucher l'idéologie et les attitudes linguistiques aux XVIIe et XVIII siècles. La recherche comparative effectuée ici sur un corpus de journaux historiques semble indiquer que le lien entre la presse et les idéologies linguistiques date du tout début des publications périodiques. Même si nous avons identifié des différences entre les trois sous-genres journalistiques analysés ici, il semble possible de parler de la présence d'une idéologie de la langue dans la presse aux siècles classique et postclassique. Cette idéologie repose premièrement sur le prestige et l'utilité de la langue, que ce soit en France ou en dehors des frontières nationales, dans les échanges diplomatiques ou encore dans le monde du livre. Une deuxième partie de l'idéologie concerne l'idéologie du standard et tout particulièrement les différents éléments de l'idéologie de la langue associés aux remarqueurs. La dernière partie concerne les institutions de la langue : le français est présenté comme une langue soutenue par l'autorité exceptionnelle de l'Académie française et par le modèle des grands auteurs, traducteurs et remarqueurs du siècle classique. Un lecteur contemporain ne peut ne pas être frappé par la familiarité de ces trois aspects de l'image de la langue qui est présentée par la presse historique des XVIIe et XVIII siècles. Il y a pourtant un aspect de l'idéologie qui manque, à savoir celui qui concerne les attitudes envers les différentes variétés de la langue. Le fait de renforcer l'importance du bon usage signale le rejet des autres variétés de manière implicite mais on ne trouve presque aucun commentaire sur les variétés particulières qui sont touchées par la presse contemporaine telles que la langue des jeunes.

Ce travail fondé sur notre corpus ne fait qu'ouvrir une piste de recherche concernant l'idéologie de la langue et la presse historique. Nous voulons donc terminer l'article avec quelques suggestions pour des recherches futures. Il sera d'abord nécessaire d'élargir le champ de recherche pour inclure tous les numéros de certains périodiques au lieu d'utiliser la combinaison d'un corpus sélectif et des tables analytiques que nous avons employée ici. Aussi sera-t-il nécessaire de viser des périodiques plus populaires et les périodiques non généralistes que nous avons dû exclure de la présente étude. Il sera surtout important dans d'études futures de prendre en compte la réception des périodiques pour accéder à une compréhension plus fine des effets du discours métalinguistique de la presse historique. 


\section{Références}

\section{Tables analytiques}

Journal des savans (1753-1764), Table générale des matieres contenues dans le Journal des Savans. Depuis l'année 1665. Qu'il a commencé, jusqu'en 1750. Inclusivement; avec les noms des Auteurs, les Titres de leurs Ouvrages, \& l'extrait des Jugemens qu'on en a portés, 10 vols, Paris, Briasson.

Vincent, Monique (1988), Mercure Galant. Extraordinaire, Affaires du temps. Table analytique contenant l'inventaire de tous les articles publiés 1672-1710, Paris, H. Champion.

\section{Textes du XVII'e et du XVIII ${ }^{e}$ siècles}

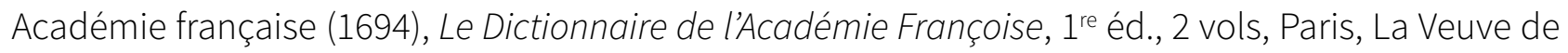
J. B. Coignard et J. B. Coignard.

Aisy, Jean d' (1698), Le Genie de la langue françoise, Paris, L. d'Houry.

Alemand, Louis-Augustin (1688), Nouvelles Observations, ou Guerre civile des François, sur la langue, Paris, J.-B. Langlois.

Bouhours, Dominique (1671), Les Entretiens d'Ariste et d'Eugène, Paris, S. Mabre-Cramoisy.

Malherbe, V. (1725), La langue françoise, expliquée dans un ordre nouveau, où l'on trouve des principes certains sur toutes les Parties du Discours. Plusieurs Lettres choisies, tirées des meilleurs Auteurs, avec des Remarques Critiques \& un Abregé de la Versification, Paris, Nicolas Le Breton.

Ménage, Gilles (1672), Observations de Monsieur Ménage sur la langue françoise, Paris, C. Barbin.

Olivet, M. l'Abbé d' (1738), Remarques de grammaire sur Racine, Paris, Gandouin.

Tallemant, Paul (1698), Remarques et decisions de l’Académie françoise, Paris, J.-B. Coignard.

Vaugelas, Claude Favre de (1647), Remarques sur la langue françoise utiles à ceux qui veulent bien parler et bien escrire, Paris, Veuve J. Camusat et P. le Petit.

Vaugelas, Claude Favre de (1690), Nouvelles Remarques de M. de Vaugelas sur la langue françoise. Ouvrage posthume. Avec des Observations de $M^{* * * \star}$, Avocat au Parlement, Paris, G. Desprez.

\section{Références secondaires}

Aquino-Weber, Dorothée, Sara Cotelli et Andres Kristol (dir.) (2009), Sociolinguistique historique du domaine gallo-roman : enjeux et méthodologies, Berne, Peter Lang.

Ayres-Bennett, Wendy (1987), Vaugelas and the development of the French language, Londres, The Modern Humanities Research Association. 
Ayres-Bennett, Wendy (1996), A history of the French language through texts, Londres/New York, Routledge.

Ayres-Bennett, Wendy (2004), Sociolinguistic variation in seventeenth-century France : methodology and case studies, Cambridge, Cambridge University Press.

Ayres-Bennett, Wendy et Magali Seijido (2011), Remarques et observations sur la langue française: histoire et évolution d'un genre, Paris, Classiques Garnier.

Ayres-Bennett, Wendy et Magali Seijido (2013), «Introduction », dans Wendy Ayres-Bennett et Magali Seijido (dir.), Bon usage et variation sociolinguistique : perspectives diachroniques et traditions nationales, Lyon, ENS Éditions, p. 7-19.

Bochnakowa, Anna (2005), Le bon français de la fin du XXe siècle : chroniques du «Figaro » 1996-2000, Cracovie, Wydawnictwo Uniwersytetu Jagiellońskiego.

Bochnakowa, Anna (2013), "Chroniques de langage dans Le Figaro (1996-2000) », dans Wendy Ayres-Bennett et Magali Seijido (dir.), Bon usage et variation sociolinguistique : perspectives diachroniques et traditions nationales, Lyon, ENS Éditions, p. 171-177.

Brownlees, Nicholas (dir.) (2006), News discourse in early modern England, Berne, Peter Lang.

Brunot, Ferdinand (1917), Histoire de la langue française des origines à 1900, vol. 5, Paris, A. Colin.

Busse, Winfried et Françoise Dougnac (1992), François-Urbain Domergue : le grammairien patriote (1745-1810), Tübingen, Gunter Narr.

Caron, Philippe (dir.) (2004), Les remarqueurs sur la langue française du XVIe siècle à nos jours, Rennes, Presses Universitaires de Rennes.

Cellard, Jacques (1983), « Les chroniques de langage », dans Edith Bédard et Jacques Maurais (dir.), La norme linguistique, Québec/Paris, Conseil de la langue française/Le Robert, p. 651-666.

Choi, E-Jung (2007), "Quels facteurs (linguistiques ou historiques) considérer dans l'accord en français? Etude de certains cas dans le Journal de la langue française (1784-1792) d'Urbain Domergue ", dans Douglas A. Kibbee (dir.), History of Linguistics 2005, Amsterdam, Benjamins, p. 183-196.

Dotoli, Giovanni (2010), Traduire en français du moyen âge au xxie siècle, Paris, Hermann.

Dougnac, Françoise (1991), « Journal de la langue française (1784-1795) », dans Jean Sgard (dir.), Dictionnaire des journaux 1600-1789, vol. 2, Oxford, Voltaire Foundation, p. 658-696.

Feyel, Gilles (1999), La presse en France des origines à 1944, Paris, Ellipses.

Feyel, Gilles (2000), L'annonce et la nouvelle : la presse d'information en France sous l'ancien régime (1630-1788), Oxford, Voltaire Foundation.

Jucker, Andreas H. (dir.) (2009), Early modern English news discourse : newspapers, pamphlets and scientific news discourse, Amsterdam/Philadelphie, J. Benjamins. 
Lodge, R. Anthony (2004), A sociolinguistic history of Parisian French, Cambridge, Cambridge University Press.

McLaughlin, Mairi (2014), « La traduction comme source de changements linguistiques dans l'histoire de la langue française », dans Wendy Ayres-Bennett et Thomas M. Rainsford (dir.), L'histoire du français. État des lieux et perspectives, Paris, Garnier, p. 273-288.

McLaughlin, Mairi (à paraître), «La traduction et l'innovation linguistique dans la presse d'information sous l'ancien régime ", dans Patricia Marie Phillips et Florence Xiangyun Zhang (dir.), Actes du colloque Translation as innovation: bridging the sciences and the humanities, Champaign (IL), Dalkey Archive Press.

Milroy, Lesley (2004), «Language ideologies and linguistic change », dans Carmen Faught (dir.), Sociolinguistic variation: critical reflexion, Oxford, Oxford University Press, p. 161-177.

Monroe, B. S. (1910), «An English academy », Modern philology, vol. 8, nº 1, p. 107-122.

Muller, Charles (2004), «Les années cinquante : l'âge d'or des remarqueurs », dans Philippe Caron (dir.), Les remarqueurs sur la langue française du XVIe siècle à nos jours, Rennes, Presses Universitaires de Rennes, p. 243-250.

Percy, Carol (2012) «Early advertising and newspapers as sources of sociolinguistic investigation », dans Juan Manuel Hernández-Campoy et Juan Camilo Conde-Silvestre (dir.), The handbook of historical sociolinguistics, Chichester, Wiley-Blackwell, p. 191-210.

Pooley, Tim et Dominique Largorgette (dir.) (2011), On linguistic change in French : socio-historical approaches. Studies in honour of R. Anthony Lodge, Chambéry, Université de Savoie.

Preston, Dennis R. (2013), « Language with an attitude », dans J. K. Chambers et Natalie Schilling (dir.), The handbook of language variation and change, $2^{e}$ éd., Malden (MA), Wiley-Blackwell, p. 157-182. [1 éd., 2002.]

Remysen, Wim (2005), «La chronique de langage à la lumière de l'expérience canadienne-française: un essai de définition », dans Julie Bérubé, Karine Gauvin et Wim Remysen (dir.), Les journées de linguistique. Actes du 18e colloque, 11-12 mars 2004, Québec, Centre interdisciplinaire de recherches sur les activités langagières, p. 267-281.

Remysen, Wim (2012), « Les représentations identitaires dans le discours normatif des chroniqueurs de langage canadiens-français depuis le milieu du XIXe siècle », Journal of French language studies, n²2, p. 419-444.

Sairio, Anni et Minna Palander-Collin (2012), " The reconstruction of prestige patterns in language history », dans Juan Manuel Hernández-Campoy et Juan Camilo Conde-Silvestre (dir.), The handbook of historical sociolinguistics, Chichester, Wiley-Blackwell, p. 626-638.

Seguin, Jean-Pierre (1999), «IV : la langue française aux XVIIe et XVIII siècles », dans Jacques Chaurand (dir.), Nouvelle histoire de la langue française, Paris, Éditions du Seuil, p. 227-344.

Sgard, Jean (dir.) (1991), Dictionnaire des journaux 1600-1789, 2 vol., Oxford, Voltaire Foundation. 
Siouffi, Gilles (2004), «Préface», dans Philippe Caron (dir.), Les remarqueurs sur la langue française du XVIe siècle à nos jours, Rennes, Presses Universitaires de Rennes, p. 7-16.

Siouffi, Gilles (2007), « De la renaissance à la révolution », dans Alain Rey, Frédéric Duval et Gilles Siouffi (dir.), Mille ans de langue française : histoire d'une passion, Paris, France Loisirs, p. 457-957.

Siouffi, Gilles (2010), Le génie de la langue française : études sur les structures imaginaires de la description linguistique à l'âge classique, Paris, H. Champion.

Valenzuela, Sebastián et Teresa Correa (2009), «Press coverage and public opinion on women candidates : the case of Chile's Michelle Bachelet », The international communication gazette, vol. 71, n०3, p. 203-233.

Vincent, Monique (2005), Le Mercure galant: présentation de la première revue féminine d'information et de culture, 1672-1710, Paris, H. Champion. 